Open J. Math. Anal., Vol. 1(2017), No. 2, pp. 01-19

Website: https://pisrt.org/psr-press/journals/oma/

ISSN: 2616-8111(Online), 2616-8103 (Print)

http://dx.doi.org/10.30538/psrp-oma2017.0006

\title{
MHD COUETTE AND POISEUILLE FLOW OF A THIRD GRADE FLUID
}

\author{
MUHAMMAD MOHSIN KAMRAN ${ }^{1}$, IMRAN SIDDIQUE
}

\begin{abstract}
The main theme of this work is to apply the Adomian decomposition method (ADM) to solve the non-linear differential equations which arise in fluid mechanics. we study some steady unidirectional magnetohydrodynamics (MHD) flow problems namely, Couette flow, Poiseuille flow and Generalized-Couette flow of a third grade non-Newtonian fluid between two horizontal infinite parallel plates in the presence of a transversal magnetic field. Moreover, the MHD solutions for a Newtonian fluid, as well as those corresponding to a third grade fluid are obtained by the limiting cases of our solutions. Finally, the influence of the pertinent parameters on the velocity of fluids is also analyzed by graphical illustrations.
\end{abstract}

AMS Mathematics Subject Classification: 76A05.

Key words and phrases: third grade fluid; Couette flow; poiseuille flow; generalized-Couette flow; steady flow; infinite coaxial circular cylinders.

\section{Introduction}

Due to diversity of fluids in nature, a lot of models have been proposed to describe their flow behavior in different circumstances. A wide range of commonly encountered fluids includes Newtonian fluids. But a large number of fluids appearing in industry differ greatly from Newtonian fluids in their rheology. Newtonian fluids are recognized by the linear relationship between stress and the rate of strain. In many existing fluids with complex molecular structure, the relation between stress and strain is found to be non-linear. Therefore, the Newtonian fluids model can not be used to predict, analyze and stimulate the behavior

Received 12 October 2017. Revised 12 December 2017.

${ }^{1}$ Corresponding Muhammad Mohsin Kamran

(C) 2017 Muhammad Mohsin Kamran, Imran Siddique. This is an open access article distributed under the Creative Commons Attribution License, which permits unrestricted use, distribution, and reproduction in any medium, provided the original work is properly cited. 
of many viscoelastic fluids. Hence, in practical situations and applications in industry, it is necessary to study of the flow behavior of non-Newtonian fluids. The inadequacy of the classical Navier-Stokes theory to describes the behavior of the rheologically complex fluids such as polymer solutions, heavy oils, blood and many emulsions, has led to the development of models of non-Newtonian fluids. In particular, many pastes, slurries, synovial, polymer solutions and suspensions exhibit shear thinning behavior. The non-newtonian fluids are widely used in chemical engineering, food industry, biological analysis, petroleum industry, and many other fields. The academic workers and engineers are very much interested in the geometry of flows of such types of fluids. As compared to Newtonian fluids, the analysis of the behavior of the motion of such fluids is much more complicated and not easy to handle because of non-linear relationship between stress and rate of strain.

In recent years many non-Newtonian models have been proposed. Among these models, model of "fluids of differential type," [1] have received considerable attention. "Fluid of third grade" is a subclass of of fluids of differential type, which has been studied successfully in various types of flow situations $[2,3,4]$ and is known to capture the non-Newtonian affects such as shear thinning, shear thickening as well as normal stress.

Most of the nonlinear differential equations do not have analytical solution. However researchers used many numerical methods, but these methods require much time and more efficient computing devices. Semi analytical methods are more suitable than numerical methods to solve nonlinear non-homogenous partial differential equations. The most powerful tool for the calculation of analytical solutions of the linear or nonlinear partial differential equation is Adomian's decomposition method (ADM), a method introduced by Adomian [5, 6]. The ADM provides analytical solution in the form of an infinite convergent power series in which each term can be easily determined. The ADM has been successfully applied to solve nonlinear differential equations in studying interesting problems arising in applied sciences and engineering [6, 7]. In 2009 Siddiqui, et al. [8] studied parallel plate flow of a third grade fluid by means of ADM and compare the results with num erical schemes. Recently, Siddiquie et al. [9] compare the ADM and HPM in solving the problem of squeezing flow between two circular plates. Their comparison shows that the ADM requires more computational efforts than the HPM, but it yields more accurate results than the HPM.

In the past few years, magnetohydrodynamics (MHD) has gained considerable importance because of its diverse applications in physics and engineering. In astrophysical and geophysical applications it is useful to study the stellar and solar structures solar storms and flares, radio propagation through the ionosphere etc. In engineering its applications are in MHD generators, MHD pumps and MHD bearings. The use of a MHD fluid as lubricant is of interest in industrial applications, because it prevents the unexpected variation of lubricant viscosity with temperature under certain extreme operating conditions. 
Historically Rossow [10] initiated the boundary layer flow on a semi infinite flat plate. Since then large amount of literature is developed on this subject. The recent attempts in this directions are made by Hayat et al. [11, 12, 13], Khan et al. [14]. S. Islam et al. [15], obtained series solutions for MHD flow between infinite parallel plates.

The main aim of this work is to study the steady MHD flow of incompressible third grade non-Newtonian fluid between two parallel plates separated by a finite gap in the presence of a transversal magnetic field and to study the steady flow of incompressible third grade non-Newtonian fluid between two coaxial cylinders of infinite length [16], using the ADM. Siddiqui, et al. [8] solved the three fundamental problems of plane Couette flow, fully developed plane Poiseuille flow and plane Couette-Poiseuille flow by using the ADM. We extend the work of Siddiqui [8] to the case of a MHD fluid. We apply the ADM to solve the problems modeling the MHD flow of a third grade fluid between two parallel plats separated by a finite gab in the presence of a transversal magnetic field. Specially, we study the three fundamental problems namely, plane Couette flow (flow due to motion of either of the plates), Poiseulle flow (flow due to application of external pressure gradient, while both plates are stationary), and GeneralizedCouette flow (flow due to motion of one plate as well as applied external pressure gradient). Also, the solution of ordinary third grade fluid are recovered [8] as limiting cases of our obtained solutions. At the end of this chapter, the influences of the parameters and other material constants on the velocity field are analyzed graphically.

\section{Mathematical formulation of the problem}

The basic equations which govern the incompressible unidirectional magnetohydrodynamic flow are:

$$
\begin{gathered}
\nabla \cdot \mathbf{V}=0 \\
\rho\left[\frac{\partial \mathbf{V}}{\partial t}+(\mathbf{V} \cdot \nabla) \mathbf{V}\right]=\rho \mathbf{f}+\nabla \cdot \mathbf{T}+\mathbf{J} \times \mathbf{B},
\end{gathered}
$$

where, $\mathbf{J}$ is electric current density and $\mathbf{B}$ is the total magnetic field, $\mathbf{B}=\mathbf{B}_{0}+\mathbf{b}$, $\mathbf{B}_{0}$ represents the imposed magnetic field and $\mathbf{b}$ denotes the induced magnetic field. In the absence of displacement currents, the Ohm's Law and Maxwell's equation [4] are

$$
\begin{gathered}
\mathbf{J}=\sigma[\mathbf{E}+\mathbf{V} \times \mathbf{B}], \\
\operatorname{div} \mathbf{B}=0, \nabla \times \mathbf{B}=\mu_{m} \mathbf{J}, \operatorname{curl} \mathbf{E}=-\frac{\partial \mathbf{B}}{\partial t},
\end{gathered}
$$

in which $\sigma$ is the electrical conductivity, $\mu_{m}$ is the magnetic permeability.

The following assumptions are made in order to lead our discussion,

(1) The density $\rho$, magnetic permeability $\mu_{m}$ and electrical conductivity $\sigma$ are assumed to be constant throughout the flow field region.

(2) The electrical conductivity $\sigma$ of the fluid considers being finite. 
(3) The total magnetic field $\mathbf{B}$ is perpendicular to the velocity field $\mathbf{V}$ and the induced magnetic field $\mathbf{b}$ is negligible compared with the applied magnetic field $\mathbf{B}_{0}$ so that the magnetic Reynolds number is small [6].

(4) We assume a situation where no energy is added or extracted from the fluid by the electric field, which implies that there is no electric field present in the fluid flow region.

Under these assumptions, the magnetohydrodynamic force involved in (2) takes the following form

$$
\mathbf{J} \times \mathbf{B}=\sigma\left[\mathbf{B}_{0}\left(\mathbf{V} \cdot \mathbf{B}_{0}\right)-\mathbf{V}\left(\mathbf{B}_{0} \cdot \mathbf{B}_{0}\right)\right]=-\sigma \mathbf{B}_{0}^{2} \mathbf{V} .
$$

For incompressible third grade fluid, the constitutive equation for extra stress tensor is given in [17]

For the problem under consideration we assume a velocity field for one dimensional flow and stress tensor of the form

$$
\mathbf{V}=(u(y), 0,0), \quad \mathbf{S}=S(y),
$$

By using Eq. (4), the continuity equation (1) is identically satisfied and the equation of motion (2), in the absence of gravitational effect becomes

$$
\begin{gathered}
-\frac{\partial p}{\partial x}+\mu \frac{\partial^{2} u}{\partial y^{2}}+6\left(\beta_{2}+\beta_{3}\right)\left(\frac{\partial u}{\partial y}\right)^{2} \frac{\partial^{2} u}{\partial y^{2}}-\sigma B_{0}^{2} u=0, \\
-\frac{\partial p}{\partial y}+\frac{\partial}{\partial y}\left\{\left(2 \alpha_{1}+\alpha_{2}\right)\left(\frac{\partial u}{\partial y}\right)^{2}\right\}=0 . \\
\frac{\partial p}{\partial z}=0 .
\end{gathered}
$$

Introducing the generalized pressure $\widehat{p}$

$$
\widehat{p}=-p(x, y)+\left(2 \alpha_{1}+\alpha_{2}\right)\left(\frac{\partial u}{\partial y}\right)^{2}
$$

and substituting $\widehat{p}$ in Eq. (ref6), we find that

$$
\frac{d \widehat{p}}{d y}=0,
$$

showing that $\widehat{p}=\widehat{p}(x)$. Consequently, Eq.(5) reduces to the single equation

$$
-\frac{d \widehat{p}}{d x}+\mu \frac{d^{2} u}{d y^{2}}+6 \beta\left(\frac{d u}{d y}\right)^{2} \frac{d^{2} u}{d y^{2}}-\sigma B_{0}^{2} u=0,
$$

where for simplicity we have introduced $\beta=\beta_{2}+\beta_{3}$.

Eq. (10) is a second-order nonlinear ordinary differential equation. This equations governs the unidirectional flow of a non-Newtonian third grade fluid between two infinite parallel plates. 


\section{Solution of the problem using Adomian Decomposition Method}

In this section, we use the ADM to solve Eq. (10) with boundary conditions corresponding to different problems of Couette flow.

3.1. Plane Couette Flow. We consider the steady laminar flow of an incompressible third grade fluid between two infinite parallel plate at $y=0$, and $y=h$. The plates are separated by distance $h$. The upper plate moves parallel to itself with uniform velocity $U$, while the lower one is at rest. The plates are non conducting and a transversal magmatic field is applied in the vertical upward direction. Let $\mathrm{x}$-axis be taken along the direction of flow and $y$ in the direction normal to the flow. The pressure $p$ is constant and fluid properties vary along $y$-axis only. Thus, governing equation (10) for such a flow, in the absence of pressure gradient, reduces to

$$
\frac{d^{2} u}{d y^{2}}+\frac{6 \beta}{\mu}\left(\frac{d u}{d y}\right)^{2} \frac{d^{2} u}{d y^{2}}-m^{2} u=0
$$

where, $m^{2}=\sigma B_{0}^{2} / \mu$,

subject to the boundary conditions

$$
\begin{aligned}
& u(y)=0 \text { at } y=0, \\
& u(y)=U \text { at } y=h .
\end{aligned}
$$

Introducing the following non-dimensional parameters

$$
u^{*}=\frac{u}{U}, \quad y^{*}=\frac{y}{h}, \quad \beta^{*}=\frac{\beta}{\mu h^{2} / U^{2}}, \quad m^{* 2}=\frac{\sigma B_{0}^{2}}{\mu / h^{2}} .
$$

Dropping the ${ }^{\prime} *$ ' the boundary value problem (1) becomes

$$
\frac{d^{2} u}{d y^{2}}+6 \beta\left(\frac{d u}{d y}\right)^{2} \frac{d^{2} u}{d y^{2}}-m^{2} u=0
$$

with the boundary conditions

$$
\begin{aligned}
& u(y)=0 \text { at } y=0, \\
& u(y)=1 \text { at } y=1 .
\end{aligned}
$$

In the operator form, Eq. (4) becomes

$$
L u=-6 \beta\left(\frac{d u}{d y}\right)^{2} \frac{d^{2} u}{d y^{2}}+m^{2} u
$$

where $L=d^{2} / d y^{2}$ and inverse operator is given by $\tilde{L}^{-1}=\iint(\cdot) d y d y$. Applying $\tilde{L}^{-1}$ on both sides of Eq. (6), we have

$$
\begin{gathered}
\tilde{L}^{-1} L u=-\tilde{L}^{-1}\left[6 \beta\left(\frac{d u}{d y}\right)^{2} \frac{d^{2} u}{d y^{2}}\right]+\tilde{L}^{-1}\left[m^{2} u\right], \\
u(y)=A y+B-6 \beta \tilde{L}^{-1}\left[\left(\frac{d u}{d y}\right)^{2} \frac{d^{2} u}{d y^{2}}\right]+\tilde{L}^{-1}\left[m^{2} u\right],
\end{gathered}
$$


where $A$ and $B$ are constants of integration.

To solve Eq. (8) by the ADM, we let

$$
\begin{aligned}
& u=\sum_{n=0}^{\infty} u_{n}(y), \\
& N u=\sum_{n=0}^{\infty} A_{n},
\end{aligned}
$$

where

$$
N u=\left(\frac{d u}{d y}\right)^{2} \frac{d^{2} u}{d y^{2}}
$$

In view of Eqs. (9) and (10), Eq. (8) becomes

$$
\sum_{n=0}^{\infty} u_{n}(y)=A y+B-6 \beta \tilde{L}^{-1} \sum_{n=0}^{\infty} A_{n}+m^{2} \tilde{L}^{-1} \sum_{n=0}^{\infty} u_{n}(y) .
$$

We identify the zeroth component as

$$
u_{0}(y)=A y+B,
$$

and the remaining components as the recurrence relation

$$
u_{n+1}(y)=-6 \beta \tilde{L}^{-1}\left[A_{n}\right]+m^{2} \tilde{L}^{-1}\left[u_{n}(y)\right], \quad n \geq 0,
$$

where $A_{n}$ 's are the Adomian polynomials that represent the non linear term in (11). The first few Adomian polynomials as follows:

$$
\begin{gathered}
A_{0}=\left(\frac{d u_{0}}{d y}\right)^{2} \frac{d^{2} u_{0}}{d y^{2}} \\
A_{1}=2 \frac{d u_{0}}{d y} \frac{d u_{1}}{d y} \frac{d^{2} u_{0}}{d y^{2}}+\left(\frac{d u_{0}}{d y}\right)^{2} \frac{d^{2} u_{1}}{d y^{2}} \\
A_{2}=2 \frac{d u_{0}}{d y} \frac{d^{2} u_{0}}{d y^{2}} \frac{d u_{2}}{d y}+\left(\frac{d u_{1}}{d y}\right)^{2} \frac{d^{2} u_{0}}{d y^{2}}+2 \frac{d u_{0}}{d y} \frac{d u_{1}}{d y} \frac{d^{2} u_{1}}{d y^{2}}+\left(\frac{d u_{0}}{d y}\right)^{2} \frac{d^{2} u_{2}}{d y^{2}}
\end{gathered}
$$

Using expression (9) in (5), we have the following boundary conditions

$$
\begin{aligned}
& u_{0}(y)=0 \text { at } y=0, \\
& u_{0}(y)=1 \text { at } y=1,
\end{aligned}
$$

and

$$
\begin{gathered}
u_{n}(y)=0 \text { at } y=0, \\
u_{n}(y)=0 \text { at } y=1, \quad n \geq 1 .
\end{gathered}
$$

From Eq. (23) and boundary conditions (26), we obtain the zeroth-order solution as

$$
u_{0}(y)=y,
$$


From (24), $(25)_{1}$ and (27), we have the first-order problem as

$$
u_{1}(y)=-6 \beta \tilde{L}^{-1}\left[A_{0}\right]+m^{2} \tilde{L}^{-1}\left[u_{0}\right],
$$

subject to the boundary conditions

$$
\begin{aligned}
& u_{1}(y)=0 \text { at } y=0, \\
& u_{1}(y)=0 \text { at } y=1 .
\end{aligned}
$$

Solving (29) and (30), the first-order solution is given by

$$
u_{1}(y)=\frac{m^{2}}{6}\left[y^{3}-y\right] .
$$

In view of $(24),(25)_{1,2}$ and (27), the second-order problem is

$$
u_{2}(y)=-6 \beta \tilde{L}^{-1}\left[A_{1}\right]+m^{2} \tilde{L}^{-1}\left[u_{1}\right],
$$

subject to boundary conditions

$$
\begin{aligned}
& u_{2}(y)=0 \text { at } y=0, \\
& u_{2}(y)=0 \text { at } y=1 .
\end{aligned}
$$

The corresponding solution is

$$
u_{2}(y)=-\beta m^{2}\left[y^{3}-y\right]+\frac{m^{4}}{6}\left[\frac{y^{5}}{20}-\frac{y^{3}}{6}+\frac{7 y}{60}\right] .
$$

Similarly, third-order problem is

$$
u_{3}(y)=-6 \beta \tilde{L}^{-1}\left[A_{2}\right]+m^{2} \tilde{L}^{-1}\left[u_{2}\right],
$$

with boundary conditions

$$
\begin{aligned}
& u_{3}(y)=0 \text { at } y=0, \\
& u_{3}(y)=0 \text { at } y=1 .
\end{aligned}
$$

The corresponding third-order solution is given by

$u_{3}(y)=6 m^{2} \beta^{2}\left[y^{3}-y\right]-\beta m^{4}\left[\frac{2 y^{5}}{5}-\frac{2 y^{3}}{3}+\frac{4 y}{15}\right]+\frac{m^{6}}{6}\left[\frac{y^{7}}{840}-\frac{y^{5}}{120}+\frac{7 y^{3}}{360}-\frac{31 y}{2520}\right]$.

Inserting (18), (21), (24) and (27) in (9), the solution of differential equation (1) takes the form

$$
u(y)=\sum_{n=0}^{\infty} u_{n}(y)=u_{0}(y)+u_{1}(y)+u_{2}(y)+u_{3}(y)+\cdots
$$

or, equivalently

$$
\begin{gathered}
u(y)=y+\frac{m^{2}}{6}\left[y^{3}-y\right]-\beta m^{2}\left[y^{3}-y\right]+6 \beta^{2} m^{2}\left[y^{3}-y\right] \\
-\beta m^{4}\left[\frac{2 y^{5}}{5}-\frac{2 y^{3}}{3}+\frac{4 y}{15}\right]+\frac{m^{4}}{6}\left[\frac{y^{5}}{20}-\frac{y^{3}}{6}+\frac{7 y}{60}\right]+\frac{m^{6}}{6}\left[\frac{y^{7}}{840}-\frac{y^{5}}{120}+\frac{7 y^{3}}{360}-\frac{31 y}{2520}\right] .
\end{gathered}
$$


It represents the velocity field for the MHD flow of a non-Newtonian third grade fluid between two parallel plates. By taking $m=0$ in Eq. (38) we recover the same solution as obtained in [8].

3.2. Fully developed plane Poiseuille Flow. For fully developed Poiseuille Flow, we consider the steady laminar flow of third grade fluid between two stationary infinite parallel plats under constant pressure gradient. Let the separation between two plates is $2 h$ and the plates are at $y=h$ and $y=-h$. Thus, the governing equation (10) in the presence of the constant pressure gradient and transversal magnetic field takes the form

$$
\frac{d^{2} u}{d y^{2}}+\frac{6 \beta}{\mu}\left(\frac{d u}{d y}\right)^{2} \frac{d^{2} u^{2}}{d y^{2}}-m^{2} u=\frac{1}{\mu} \frac{d \widehat{p}}{d x}
$$

with the boundary conditions

$$
\begin{gathered}
u(y)=0 \text { at } y=h, \\
u(y)=0 \text { at } y=-h,
\end{gathered}
$$

Introducing the non-dimensional parameters

$$
u^{*}=\frac{u}{U}, \quad y^{*}=\frac{y}{h}, \quad \beta^{*}=\frac{\beta U^{2}}{\mu h^{2}}, \quad x^{*}=\frac{x}{h}, \quad p^{*}=\frac{\widehat{p}}{\mu U / h}, \quad m^{* 2}=\frac{\sigma B_{0}^{2}}{\mu / h^{2}},
$$

Eqs. (39) and (40) after dropping ' ${ }^{\prime}$ take the following form:

$$
\begin{gathered}
\frac{d^{2} u}{d y^{2}}+6 \beta\left(\frac{d u}{d y}\right)^{2} \frac{d^{2} u}{d y^{2}}-m^{2} u=\frac{d p}{d x}, \\
u(y)=0 \text { at } y=1, \\
u(y)=0 \text { at } y=-1 .
\end{gathered}
$$

Let us apply the ADM to solve the above boundary value problem. Accordingly, in operator form Eq. (42) becomes

$$
L u=\frac{d p}{d x}-6 \beta\left[\left(\frac{d u}{d y}\right)^{2} \frac{d^{2} u}{d y^{2}}\right]+\left[m^{2} u\right]
$$

where $L=d^{2} / d y^{2}$, and $N u=(d u / d y)^{2} d^{2} u / d y^{2}$ are linear and nonlinear terms respectively.

Applying the inverse operator $\tilde{L}^{-1}=\iint(\cdot) d y d y$ on both sides to equation (44), we get

$$
\tilde{L}^{-1} L u=\tilde{L}^{-1}\left(\frac{d p}{d x}\right)-6 \beta \tilde{L}^{-1}\left[\left(\frac{d u}{d y}\right)^{2} \frac{d^{2} u}{d y^{2}}\right]+\tilde{L}^{-1}\left[m^{2} u\right] .
$$

In view of Eqs. (9) and (10), Eq. (45) becomes

$$
\sum_{n=0}^{\infty} u_{n}(y)=A y+B+\tilde{L}^{-1}\left(\frac{d p}{d x}\right)-6 \beta \tilde{L}^{-1} \sum_{n=0}^{\infty} A_{n}+m^{2} \tilde{L}^{-1} \sum_{n=0}^{\infty} u_{n}(y) .
$$


From expression (46), we have the following recurrence relations for zeroth and higher order solutions respectively

$$
\begin{gathered}
u_{0}(y)=A y+B+\tilde{L}^{-1}\left(\frac{d p}{d x}\right), \\
u_{n+1}(y)=-6 \beta \tilde{L}^{-1}\left[A_{n}\right]+m^{2} \tilde{L}^{-1}\left[u_{n}(y)\right], n \geq 0,
\end{gathered}
$$

Using expression (9), the boundary conditions (43) becomes

$$
\begin{gathered}
u_{n}(y)=0 \text { at } y=1, \\
u_{n}(y)=0 \text { at } y=-1 .
\end{gathered}
$$

From equation (79) and (49), we have the Zeroth-order problem as

$$
u_{0}(y)=A y+B+\tilde{L}^{-1}\left(\frac{d p}{d x}\right)
$$

with the boundary conditions

$$
\begin{gathered}
u_{0}(y)=0 \text { at } y=1, \\
u_{0}(y)=0 \text { at } y=-1 .
\end{gathered}
$$

The corresponding zeroth-order solution is given by

$$
u_{0}(y)=\frac{d p}{d x}\left[\frac{y^{2}}{2}-\frac{1}{2}\right]
$$

In view of Eqs. (25), (48) and (49), we get following problems of different orders with corresponding boundary conditions as:

The first-order problem is

$$
u_{1}(y)=-6 \beta \tilde{L}^{-1}\left[A_{0}\right]+m^{2} \tilde{L}^{-1}\left[u_{0}(y)\right],
$$

subject to boundary conditions

$$
\begin{gathered}
u_{1}(y)=0 \text { at } y=1, \\
u_{1}(y)=0 \text { at } y=-1 .
\end{gathered}
$$

The corresponding solution is

$$
u_{1}(y)=-2 \beta\left(\frac{d p}{d x}\right)^{3}\left[\frac{y^{4}}{4}-\frac{1}{4}\right]+\left(\frac{d p}{d x}\right)\left[\frac{m^{2}}{2}\left(\frac{y^{4}}{12}-\frac{y^{2}}{2}+\frac{5}{12}\right)\right] .
$$

The second-order problem is

$$
u_{2}(y)=-6 \beta \tilde{L}^{-1}\left[A_{1}\right]+m^{2} \tilde{L}^{-1}\left[u_{1}(y)\right],
$$

with the boundary conditions

$$
\begin{gathered}
u_{2}(y)=0 \text { at } y=1, \\
u_{2}(y)=0 \text { at } y=-1 .
\end{gathered}
$$

The corresponding second-order solution is

$$
u_{2}(y)=3(-2 \beta)^{2}\left(\frac{d p}{d x}\right)^{5}\left[\frac{y^{6}}{6}-\frac{1}{6}\right]+(-2 \beta)\left(\frac{d p}{d x}\right)^{3}\left[m^{2}\left(\frac{11 y^{6}}{120}-\frac{3 y^{4}}{8}-\frac{y^{2}}{8}+\frac{49}{120}\right)\right]
$$




$$
+\left(\frac{d p}{d x}\right)\left[\frac{m^{4}}{2}\left(\frac{y^{6}}{360}-\frac{y^{4}}{24}+\frac{5 y^{2}}{24}-\frac{61}{360}\right)\right] .
$$

The third-order problem is

$$
u_{3}(y)=-6 \beta \tilde{L}^{-1}\left[A_{2}\right]+m^{2} \tilde{L}^{-1}\left[u_{2}(y)\right],
$$

with the boundary conditions

$$
\begin{gathered}
u_{3}(y)=0 \text { at } y=1, \\
u_{3}(y)=0 \text { at } y=-1 .
\end{gathered}
$$

The corresponding third-order solution is given by

$$
\begin{gathered}
u_{3}(y)=12(-2 \beta)^{3}\left(\frac{d p}{d x}\right)^{7}\left[\frac{y^{8}}{8}-\frac{1}{8}\right]+3(-2 \beta)^{2}\left(\frac{d p}{d x}\right)^{5}\left[m ^ { 2 } \left(\frac{127 y^{8}}{1120}-\frac{25 y^{6}}{60}-\frac{y^{4}}{16}\right.\right. \\
\left.\left.-\frac{y^{2}}{12}+\frac{503}{1120}\right)\right]+(-2 \beta)\left(\frac{d p}{d x}\right)^{3}\left[m^{4}\left(\frac{17 y^{8}}{1120}-\frac{11 y^{6}}{80}+\frac{y^{4}}{3}+\frac{49 y^{2}}{240}-\frac{93}{224}\right)\right] \\
+\left(\frac{d p}{d x}\right)\left[\frac{m^{6}}{2}\left(\frac{y^{8}}{20160}-\frac{y^{6}}{720}+\frac{5 y^{4}}{288}-\frac{61 y^{2}}{720}+\frac{277}{4032}\right)\right]
\end{gathered}
$$

Inserting (52), (55), (58) and (61) in (9) we obtain the fourth order approximate solution for a fully developed plan poiseuille MHD flow of a non-Newtonian third grade fluid.

$$
\begin{gathered}
u(y)=\frac{d p}{d x}\left[\frac{y^{2}}{2}-\frac{1}{2}\right]-2 \beta\left(\frac{d p}{d x}\right)^{3}\left[\frac{y^{4}}{4}-\frac{1}{4}\right]+3(-2 \beta)^{2}\left(\frac{d p}{d x}\right)^{5}\left[\frac{y^{6}}{6}-\frac{1}{6}\right] \\
+12(-2 \beta)^{3}\left(\frac{d p}{d x}\right)^{7} \\
{\left[\frac{y^{8}}{8}-\frac{1}{8}\right]+\left(\frac{d p}{d x}\right)\left[\frac{m^{2}}{2}\left(\frac{y^{4}}{12}-\frac{y^{2}}{2}+\frac{5}{12}\right)\right]+(-2 \beta)\left(\frac{d p}{d x}\right)^{3}\left[m ^ { 2 } \left(\frac{11 y^{6}}{120}-\frac{3 y^{4}}{8}\right.\right.} \\
\left.\left.-\frac{y^{2}}{8}+\frac{49}{120}\right)\right]+3(-2 \beta)^{2}\left(\frac{d p}{d x}\right)^{5}\left[m^{2}\left(\frac{127 y^{8}}{1120}-\frac{25 y^{6}}{60}-\frac{y^{4}}{16}-\frac{y^{2}}{12}+\frac{503}{1120}\right)\right] \\
+\left(\frac{d p}{d x}\right)\left[\frac{m^{4}}{2}\left(\frac{y^{6}}{360}-\frac{y^{4}}{24}+\frac{5 y^{2}}{24}-\frac{61}{360}\right)\right]+(-2 \beta)\left(\frac{d p}{d x}\right)^{3}\left[m ^ { 4 } \left(\frac{17 y^{8}}{1120}-\frac{11 y^{6}}{80}\right.\right. \\
\left.\left.+\frac{y^{4}}{3}+\frac{49 y^{2}}{240}-\frac{93}{224}\right)\right]+\left(\frac{d p}{d x}\right)\left[\frac{m^{6}}{2}\left(\frac{y^{8}}{20160}-\frac{y^{6}}{720}+\frac{5 y^{4}}{288}-\frac{61 y^{2}}{720}+\frac{277}{4032}\right)\right] .
\end{gathered}
$$

By taking $m=0$ in the above expression we recover the same solution as in [Eq. $2.2 .52,8]$. When we take $\beta=0$ and $m=0$ we have the exact solution for the viscous Newtonian fluid. 
3.3. MHD Generalized-Couette Flow. We now again consider the steady laminar fully developed flow of a third grade fluid between two infinite horizontal parallel plates at a distance $h$ apart. The plates are non conducting and a transversal magmatic field is applied in the vertical upward direction. The motion of the fluid is due to the motion of upper plate of uniform velocity $U$ and constant pressure gradient along $x$ direction, while the lower plate is at rest. The resulting differential equation (39) and the corresponding boundary conditions for this flow are given as respectively

$$
\begin{gathered}
\frac{d^{2} u}{d y^{2}}+\frac{6 \beta}{\mu}\left(\frac{d u}{d y}\right)^{2} \frac{d^{2} u^{2}}{d y^{2}}-m^{2} u=\frac{1}{\mu} \frac{d \widehat{p}}{d x} \\
u(y)=0 \text { at } y=0 \\
u(y)=U \text { at } y=h .
\end{gathered}
$$

Introducing the non-dimensional parameters (41), Eqs. (63) and (54) becomes

$$
\begin{gathered}
\frac{d^{2} u}{d y^{2}}+6 \beta\left(\frac{d u}{d y}\right)^{2} \frac{d^{2} u}{d y^{2}}-m^{2} u=\frac{d p}{d x}, \\
u(y)=0 \text { at } y=0 \\
u(y)=1 \text { at } y=1 .
\end{gathered}
$$

Follow the same procedure as in previous section, we define linear operator $L=d^{2} / d y^{2}$ for Eq. (65) and apply the inverse operator $\tilde{L}^{-1}=\iint(\cdot) d y d y$ on both sides to equation (55) we get

$$
u(y)=A y+B+\tilde{L}^{-1}\left(\frac{d p}{d x}\right)-6 \beta \tilde{L}^{-1}\left[\left(\frac{d u}{d y}\right)^{2} \frac{d^{2} u}{d y^{2}}\right]+\tilde{L}^{-1}\left[m^{2} u\right],
$$

where $\mathrm{A}$ and $\mathrm{B}$ are constants of integration.

In view of decomposition series (9) and (10), Eq. (67) gives

$$
\sum_{n=0}^{\infty} u_{n}(y)=A y+B+\tilde{L}^{-1}\left(\frac{d p}{d x}\right)-6 \beta \tilde{L}^{-1} \sum_{n=0}^{\infty} A_{n}+m^{2} \tilde{L}^{-1} \sum_{n=0}^{\infty} u_{n}(y),
$$

From (68) one obtains the recurrence relations

$$
\begin{gathered}
u_{0}(y)=A y+B+\tilde{L}^{-1}\left(\frac{d p}{d x}\right), \\
u_{n+1}(y)=-6 \beta \tilde{L}^{-1}\left[A_{n}\right]+m^{2} \tilde{L}^{-1}\left[u_{n}(y)\right], n \geq 0 .
\end{gathered}
$$

Using decomposition series (9) into (66), we have the following boundary conditions

and

$$
\begin{aligned}
& u_{0}(y)=0 \text { at } y=0 \\
& u_{0}(y)=1 \text { at } y=1 .
\end{aligned}
$$

$$
\begin{gathered}
u_{n}(y)=0 \text { at } y=0, \\
u_{n}(y)=0 \text { at } y=1, \quad n \geq 1 .
\end{gathered}
$$


From Eq. (69) and boundary conditions (71), we obtain the zeroth-order solution as

$$
u_{0}(y)=y+\alpha\left[y^{2}-y\right] .
$$

In view of recurrence relations (70) along with the Adomian polynomials (25), we get the following problems of different orders with corresponding boundary conditions (72) as:

The first-order problem is

$$
u_{1}(y)=-6 \beta \tilde{L}^{-1}\left[A_{0}\right]+m^{2} \tilde{L}^{-1}\left[u_{0}\right],
$$

subject to the boundary conditions

$$
\begin{aligned}
& u_{1}(y)=0 \text { at } y=0, \\
& u_{1}(y)=0 \text { at } y=1 .
\end{aligned}
$$

Solving (74) and (80), the first-order solution is given by

$$
\begin{gathered}
u_{1}(y)=\frac{-\beta}{4 \alpha}[1+\alpha(2 y-1)]^{4}+y\left[\frac{\beta}{4 \alpha}(\bar{A}-\bar{B})\right]+\left(\frac{\beta}{4 \alpha} \bar{B}\right) \\
+m^{2}\left[\alpha\left(\frac{y^{4}}{12}-\frac{y^{3}}{6}+\frac{y}{12}\right)+\frac{y^{3}}{3}-\frac{y}{3}\right],
\end{gathered}
$$

The second-order problem is

$$
u_{2}(y)=-6 \beta \tilde{L}^{-1}\left[A_{1}\right]+m^{2} \tilde{L}^{-1}\left[u_{1}(y)\right],
$$

subject to

$$
\begin{aligned}
& u_{2}(y)=0 \text { at } y=0, \\
& u_{2}(y)=0 \text { at } y=1 .
\end{aligned}
$$

The second-order solution is

$$
\begin{gathered}
u_{2}(y)=\frac{\beta^{2}}{\alpha}[1+\alpha(2 y-1)]^{6}-\left(\frac{\beta}{2 \alpha}\right)^{2}(\bar{A}-\bar{B})[1+\alpha(2 y-1)]^{3}-\left(\frac{m^{2} \beta}{480 \alpha^{3}}\right) \\
{[1+\alpha(2 y-1)]^{6}+\left(\frac{m^{2} \beta}{24 \alpha}\right)\left[(\bar{A}-\bar{B}) y^{3}\right]+\left(\frac{m^{2} \beta}{8 \alpha}\right)\left[\bar{B} y^{2}\right]+6 m^{2}(-\beta)\left[\frac{y^{3}}{6}\right.} \\
\left.+\frac{5 \alpha y^{4}}{12}-\frac{\alpha y^{3}}{2}+\frac{2 \alpha^{2} y^{5}}{5}+\frac{2 \alpha^{3} y^{6}}{15}-\frac{2 \alpha^{3} y^{5}}{5}+\frac{\alpha^{2} y^{3}}{2}+\frac{5 \alpha^{3} y^{4}}{12}-\frac{\alpha^{3} y^{3}}{6}-\frac{5 \alpha^{2} y^{4}}{6}\right] \\
+24 m^{2}(-\alpha \beta)\left[\frac{y^{4}}{24}+\frac{\alpha y^{5}}{15}-\frac{\alpha y^{4}}{12}-\frac{y^{2}}{12}+\frac{\alpha y^{2}}{8}+\frac{\alpha^{2} y^{6}}{45}-\frac{\alpha^{2} y^{5}}{15}-\frac{\alpha y^{3}}{18}+\frac{\alpha^{2} y^{3}}{36}\right. \\
\left.+\frac{\alpha^{2} y^{4}}{24}-\frac{\alpha^{2} y^{2}}{24}\right]+m^{4}\left[\frac{y^{5}}{120}+\frac{\alpha y^{6}}{360}-\frac{\alpha y^{5}}{120}-\frac{y^{3}}{36}+\frac{\alpha y^{3}}{72}\right]+\frac{\beta^{2}}{\alpha} y\left[(1-\alpha)^{6}-(1\right. \\
\left.+\alpha)^{6}\right]+\left(\frac{\beta}{2 \alpha}\right)^{2} y(\bar{A}-\bar{B})\left[(1+\alpha)^{3}-(1-\alpha)^{3}\right]+\left(\frac{m^{2} \beta}{480 \alpha^{3}}\right) y\left[(1+\alpha)^{6}\right. \\
\left.-(1-\alpha)^{6}\right]-6 m^{2}(-\beta) y\left[\frac{1}{6}-\frac{\alpha}{12}+\frac{9 \alpha^{2}}{10}-\frac{5 \alpha^{2}}{6}-\frac{\alpha^{3}}{60}\right]-24 m^{2}(-\alpha \beta)
\end{gathered}
$$




$$
\begin{gathered}
y\left[\frac{-1}{24}+\frac{19 \alpha}{360}-\frac{\alpha^{2}}{60}\right]-m^{4} y\left[\frac{-7}{360}+\frac{\alpha}{120}\right]-\left(\frac{m^{2} \beta}{8 \alpha}\right)[\bar{B} y]-\left(\frac{m^{2} \beta}{24 \alpha}\right) y(\bar{A}-\bar{B}) \\
-\left(\frac{\beta^{2}}{\alpha}\right)\left[(1-\alpha)^{6}\right]+\left(\frac{\beta}{2 \alpha}\right)^{2}(\bar{A}-\bar{B})(1-\alpha)^{3}+\left(\frac{m^{2} \beta}{480 \alpha^{3}}\right)(1-\alpha)^{6} .
\end{gathered}
$$

Summarizing these solutions, we get final form of solution as

$$
\begin{aligned}
& u(y)=y+\alpha\left[y^{2}-y\right]+\frac{-\beta}{4 \alpha}[1+\alpha(2 y-1)]^{4}+y\left[\frac{\beta}{4 \alpha}(\bar{A}-\bar{B})\right]+\left(\frac{\beta}{4 \alpha} \bar{B}\right) \\
& +m^{2}\left[\alpha\left(\frac{y^{4}}{12}-\frac{y^{3}}{6}+\frac{y}{12}\right)+\frac{y^{3}}{3}-\frac{y}{3}\right]+\frac{\beta^{2}}{\alpha}[1+\alpha(2 y-1)]^{6} \\
& -\left(\frac{\beta}{2 \alpha}\right)^{2}(\bar{A}-\bar{B})[1+\alpha(2 y-1)]^{3}-\left(\frac{m^{2} \beta}{480 \alpha^{3}}\right)[1+\alpha(2 y-1)]^{6}+\left(\frac{m^{2} \beta}{24 \alpha}\right)\left[(\bar{A}-\bar{B}) y^{3}\right] \\
& +\left(\frac{m^{2} \beta}{8 \alpha}\right)\left[\bar{B} y^{2}\right]+6 m^{2}(-\beta) \\
& {\left[\frac{y^{3}}{6}+\frac{5 \alpha y^{4}}{12}-\frac{\alpha y^{3}}{2}+\frac{2 \alpha^{2} y^{5}}{5}+\frac{2 \alpha^{3} y^{6}}{15}-\frac{2 \alpha^{3} y^{5}}{5}+\frac{\alpha^{2} y^{3}}{2}+\frac{5 \alpha^{3} y^{4}}{12}-\frac{\alpha^{3} y^{3}}{6}-\frac{5 \alpha^{2} y^{4}}{6}\right]} \\
& +24 m^{2}(-\alpha \beta) \\
& {\left[\frac{y^{4}}{24}+\frac{\alpha y^{5}}{15}-\frac{\alpha y^{4}}{12}-\frac{y^{2}}{12}+\frac{\alpha y^{2}}{8}+\frac{\alpha^{2} y^{6}}{45}-\frac{\alpha^{2} y^{5}}{15}-\frac{\alpha y^{3}}{18}+\frac{\alpha^{2} y^{3}}{36}+\frac{\alpha^{2} y^{4}}{24}-\frac{\alpha^{2} y^{2}}{24}\right]} \\
& +m^{4}\left[\frac{y^{5}}{120}+\frac{\alpha y^{6}}{360}-\frac{\alpha y^{5}}{120}-\frac{y^{3}}{36}+\frac{\alpha y^{3}}{72}\right] \\
& +\frac{\beta^{2}}{\alpha} y\left[(1-\alpha)^{6}-(1+\alpha)^{6}\right]+\left(\frac{\beta}{2 \alpha}\right)^{2} y(\bar{A}-\bar{B})\left[(1+\alpha)^{3}-(1-\alpha)^{3}\right] \\
& +\left(\frac{m^{2} \beta}{480 \alpha^{3}}\right) y\left[(1+\alpha)^{6}-(1-\alpha)^{6}\right]-6 m^{2}(-\beta) y\left[\frac{1}{6}-\frac{\alpha}{12}+\frac{9 \alpha^{2}}{10}-\frac{5 \alpha^{2}}{6}-\frac{\alpha^{3}}{60}\right]-24 m^{2}(-\alpha \beta) \\
& y\left[\frac{-1}{24}+\frac{19 \alpha}{360}-\frac{\alpha^{2}}{60}\right]-m^{4} y\left[\frac{-7}{360}+\frac{\alpha}{120}\right]-\left(\frac{m^{2} \beta}{8 \alpha}\right)[\bar{B} y]-\left(\frac{m^{2} \beta}{24 \alpha}\right) y(\bar{A}-\bar{B}) \\
& -\frac{\beta^{2}}{\alpha}(1-\alpha)^{6}+\left(\frac{\beta}{2 \alpha}\right)^{2}(\bar{A}-\bar{B})(1-\alpha)^{3}+\left(\frac{m^{2} \beta}{480 \alpha^{3}}\right)(1-\alpha)^{6} .
\end{aligned}
$$

By taking $m=0$ in the above expression we recover the same solution as obtained in [Eq. 2.2.52, 8]. When we take $\beta=0$ and $m=0$ we have the exact solution for the viscous Newtonian fluid. 


\section{Conclusion}

This work is mainly concerned with the flows of third grade fluid in Cartesian and Cylindrical frame. We have discussed the velocity field corresponding to the steady, incompressible unidirectional flow of the third grade fluid between to infinite parallel plates under the influence of MHD force term have been determined by mean of Adomain decomposition method.

After lengthy computations the solution obtained in series form satisfy all the imposed initial and boundary conditions. Graphically, results shown that by increasing the strength of magnitude field. Fluid velocity gradually decreases and by varying values of parameters velocity also varies. By taking $m=0$, we get the similar results as obtained in [7].

\section{Graphical Explanation}

\subsection{Couette Flow.}

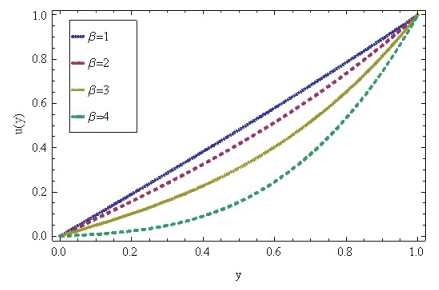

Figure 1. Profile of the dimensionless velocity for plan Couette flow with various values of non-Newtonian parameter $\beta$ and for fixed $m=1$.

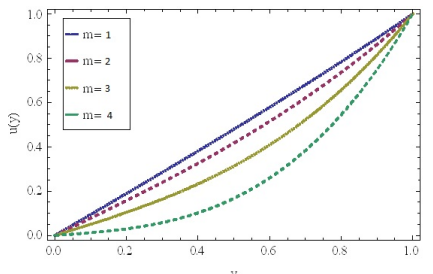

Figure 2. Profile of the dimensionless velocity for plan Couette flow with various values of the magnetic parameter for fixed value of $\beta=2$.

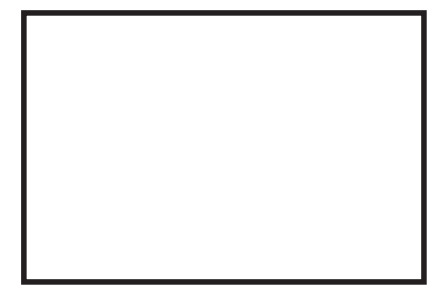

Figure 3. Comparison of PM and $\mathrm{ADM}$ at $\beta=2, m=0.1$ and $\epsilon=0.001$ 
TABLE 1. Comparison of PM and ADM

\begin{tabular}{|l|l|l|}
\hline $\mathrm{Y}$ & $\mathrm{PM}$ & $\mathrm{ADM}$ \\
\hline 0 & $-1.0829 \times 10-16$ & 0. \\
\hline 0.1 & 0.0999339 & 0.07805 \\
\hline 0.2 & 0.199872 & 0.157431 \\
\hline 0.3 & 0.299818 & 0.239473 \\
\hline 0.4 & 0.399776 & 0.325507 \\
\hline 0.5 & 0.49975 & 0.416863 \\
\hline 0.6 & 0.599744 & 0.514871 \\
\hline 0.7 & 0.699762 & 0.62086 \\
\hline 0.8 & 0.799808 & 0.73616 \\
\hline 0.9 & 0.899886 & 0.862097 \\
\hline 1 & 1 & 1 \\
\hline
\end{tabular}

\subsection{Fully developed plane Poiseuille Flow.}

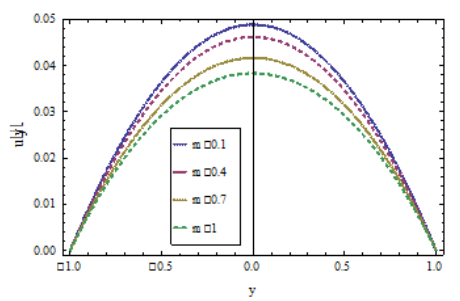

Figure 4. Profile of the dimensionless velocity $u(y)$ for fully developed plan-Poiseuille flow with various values of non-Newtonian parameter, for fixed values of $m=1$ and $p=0.1$

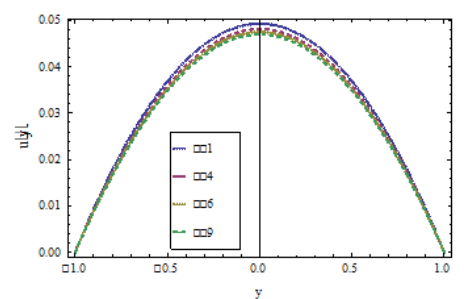

Figure 5. Profile of the dimensionless velocity $u(y)$ for fully developed plan-Poiseuille flow with various values of $p=0.1$ and for fixed values of $m=1$ and $p=2$ 


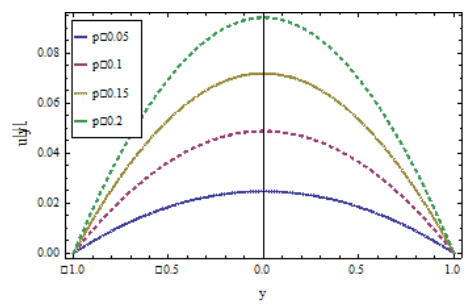

Figure 6. Profile of the dimensionless velocity $u(y)$ for fully developed plan-Poiseuille flow with various values of the magnetic parameter $m$ for fixed values $\beta=2$ and $p=0.1$

TABLE 2. Comparison of PM and ADM

\begin{tabular}{|l|l|l|}
\hline $\mathrm{Y}$ & $\mathrm{PM}$ & $\mathrm{ADM}$ \\
\hline 0 & 0.049792 & 0.0488888 \\
\hline 0.1 & 0.0492945 & 0.0483914 \\
\hline 0.2 & 0.0478019 & 0.0469001 \\
\hline 0.3 & 0.0453141 & 0.0444184 \\
\hline 0.4 & 0.0418308 & 0.0409517 \\
\hline 0.5 & 0.0373517 & 0.0365076 \\
\hline 0.6 & 0.0318763 & 0.0310954 \\
\hline 0.7 & 0.0254042 & 0.0247259 \\
\hline 0.8 & 0.0179346 & 0.0174114 \\
\hline 0.9 & 0.00946679 & 0.00916494 \\
\hline 1 & 0 & 0 \\
\hline
\end{tabular}

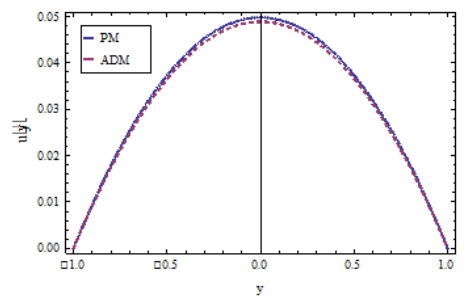

Figure 7. Comparison of $\mathrm{PM}$ and $\mathrm{ADM}$ at $\beta=2, m=0.1$ and $\epsilon=0.001$, $p=0.1$ 


\subsection{MHD Generalized-Couette Flow:}

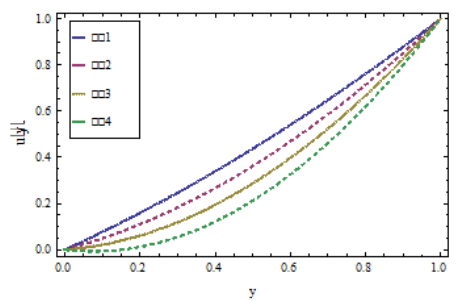

Figure 8. Profile of the dimensionless velocity $u(y)$ for fully developed plan-Poiseuille flow with various values of the magnetic parameter $\beta$ for fixed values $m=0.1$ and $p=0.1$

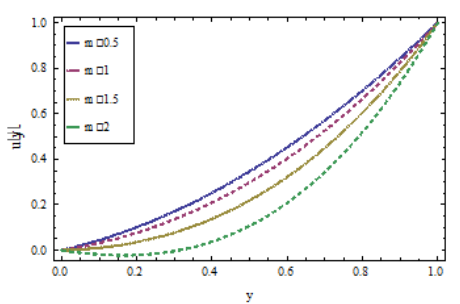

Figure 9. Profile of the dimensionless velocity $u(y)$ for generalized Couette flow with various values of $p$ and for fixed values of $m=1$ and $p=2$

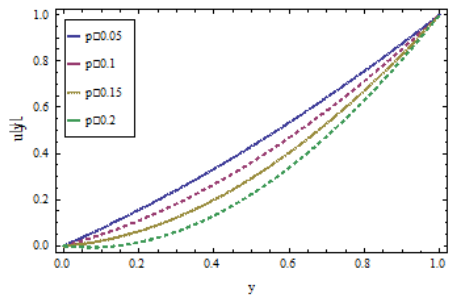

Figure 10. Profile of the dimensionless velocity $u(y)$ for generalized Couette flow with various values of the magnetic parameter $m$, for fixed values of $\beta=2$ and $p=0.1$

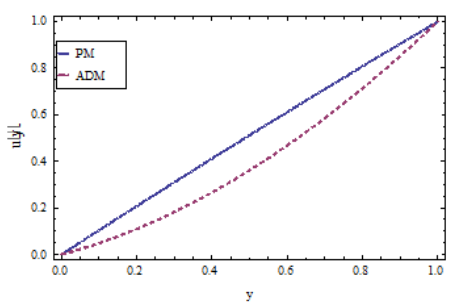

Figure 11.Comparison of $\mathrm{PM}$ and $\mathrm{ADM}$ at $\beta=2, m=0.1$ and $\epsilon=0.001$,

$$
p=0.1
$$


TABLE 3. Comparison of PM and ADM

\begin{tabular}{|l|l|l|}
\hline $\mathrm{Y}$ & $\mathrm{PM}$ & $\mathrm{ADM}$ \\
\hline 0 & $-1.73599 \times 10^{-14}$ & 0. \\
\hline 0.1 & 0.104304 & 0.048854 \\
\hline 0.2 & 0.207626 & 0.109698 \\
\hline 0.3 & 0.309974 & 0.182294 \\
\hline 0.4 & 0.411359 & 0.266405 \\
\hline 0.5 & 0.511791 & 0.361799 \\
\hline 0.6 & 0.611281 & 0.468245 \\
\hline 0.7 & 0.709836 & 0.585514 \\
\hline 0.8 & 0.807468 & 0.713378 \\
\hline 0.9 & 0.904186 & 0.851614 \\
\hline 1 & 1 & 1 \\
\hline
\end{tabular}

\section{Competing Interests}

The author(s) do not have any competing interests in the manuscript.

\section{REFERENCES}

1. Truesdell, C., \& Noll, W. (2004). The non-linear field theories of mechanics. In The nonlinear field theories of mechanics. Springer Berlin Heidelberg.

2. Rajagopal, K. R. (1980). Stability of third-grade fluids. ARCHIVES MECH., 32(6), 867875 .

3. Fosdick, R. L., \& Rajagopal, K. R. (1980, January). Thermodynamics and stability of fluids of third grade. In Proceedings of the Royal Society of London A: Mathematical, Physical and Engineering Sciences (Vol. 369, No. 1738, 351-377). The Royal Society.

4. El-Shehawey, E. F., Elbarbary, E. M., Afifi, N. A. S., \& Elshahed, M. (2000). MHD flow of an elastico-viscous fluid under periodic body acceleration. International Journal of Mathematics and Mathematical Sciences, 23(11), 795-799.

5. Cherruault, Y., \& Adomian, G. (1993). Decomposition methods: a new proof of convergence. Mathematical and Computer Modelling, 18(12), 103-106.

6. Bildik, N., \& Bayramoglu, H. (2005). The solution of two dimensional nonlinear differential equation by the Adomian decomposition method. Applied mathematics and computation, 163(2), 519-524.

7. Pamuk, S. (2005). Solution of the porous media equation by Adomian's decomposition method. Physics Letters A, 344(2), 184-188.

8. Siddiqui, A. M., Hameed, M., Siddiqui, B. M., \& Ghori, Q. K. (2010). Use of Adomian decomposition method in the study of parallel plate flow of a third grade fluid. Communications in Nonlinear Science and Numerical Simulation, 15(9), 2388-2399.

9. Siddiqui, A. M., Haroon, T., Bhatti, S., \& Ansari, A. R. (2010). A comparison of the adomian and homotopy perturbation methods in solving the problem of squeezing flow between two circular plates. Mathematical Modelling and Analysis, 15(4), 491-504.

10. Rossow, V. J. (1958). On flow of electrically conducting fluids over a flat plate in the presence of a transverse magnetic field.

11. Hayat, T., Hutter, K., Asghar, S., \& Siddiqui, A. M. (2002). MHD flows of an Oldroyd-B fluid. Mathematical and Computer Modelling, 36(9-10), 987-995. 
12. Hayat, T., Haroon, T., Asghar, S., \& Siddiqui, A. M. (2003). MHD flow of a third-grade fluid due to eccentric rotations of a porous disk and a fluid at infinity. International journal of non-linear mechanics, 38(4), 501-511.

13. Hayat, T., Khan, M., \& Ayub, M. (2004). Couette and Poiseuille flows of an Oldroyd 6 -constant fluid with magnetic field. Journal of mathematical analysis and applications, 298(1), 225-244.

14. Khan, M., Fetecau, C., \& Hayat, T. (2007). MHD transient flows in a channel of rectangular cross-section with porous medium. Physics letters A, 369(1), 44-54.

15. Islam, S. (2010). Homotopy perturbations analysis of couette and poiseuille flows of a third-grade fluid with magnetic field. Science International, 22(3).

16. Fetecau, C., \& Fetecau, C. (2006). Starting solutions for the motion of a second grade fluid due to longitudinal and torsional oscillations of a circular cylinder. International Journal of Engineering Science, 44(11), 788-796.

17. Dunn, J. E., \& Rajagopal, K. R. (1995). Fluids of differential type: critical review and thermodynamic analysis. International Journal of Engineering Science, 33(5), 689-729.

Muhammad Mohsin Kamran

Division of Science and Technology, University of Education, Lahore 54000, Pakistan.

e-mail: mohsin.kamran@ue.edu.pk

\section{Imran Saddique}

Department of Mathematics, School of Sciences, University of Management and Technology, Lahore-54000, Pakistan.

e-mail: imransiddique@umt.edu.pk 